\title{
Coherent Structures in Scalar Feed-Forward Chains
}

\author{
Christopher Browne* \& Andrew L. Dickerson ${ }^{\dagger}$ \\ Sponsors: Grégory Faye \& Arnd Scheel $^{\S}$
}

\begin{abstract}
We study semi-infinite and bi-infinite scalar feed-forward networks. We find that the temporal dynamics of these systems is closely linked to the spatial dynamics of an associated interval map and show how this interval map may be used to describe stationary interfaces. Beyond stationary structures, we show that the onset of instabilities in finite networks is intimately related to the emergence of frustrated invasion fronts. These concepts are then applied to several toy models, whose intracellular dynamics mimic the normal form of elementary steady-state bifurcations.
\end{abstract}

\section{Introduction}

We study coherent structures in scalar feed-forward networks in a systematic fashion. We focus on linear, nearest-neighbor coupling, and find that the use of spatial dynamics allows for an almost complete classification of coherent structures. Being more precise, we study systems of form

$$
\begin{aligned}
& \dot{u}_{j}=f\left(u_{j}\right)+\alpha\left(u_{j}-u_{j-1}\right), j \geq 1, \\
& \dot{u}_{0}=f\left(u_{0}\right),
\end{aligned}
$$

where $u_{j} \in \mathbb{R}$. We will also extend our analysis to bi-infinite chains where we will take $j \in \mathbb{Z}$. Throughout this paper, we explore three types of nonlinearities,

- fold: $f(u)=1-u^{2}$;

- pitchfork: $f(u)=u\left(1-u^{2}\right)$;

- cusp: $f(u)=u(1-u)(u-a)$,

which govern the intracellular dynamics of our system. The nonlinearities can be thought of as rescaled normal forms of bifurcations at individual cells. In fact, assuming weak coupling strength $\mathrm{O}(\varepsilon)$ between cells and a fold singularity in the intracellular dynamics,

$$
v_{j}^{\prime}=\varepsilon^{2}-v_{j}^{2}+\varepsilon \alpha\left(v_{j}-v_{j-1}\right)+\mathrm{O}\left(\left|\varepsilon^{2} v\right|+|v|^{3}\right),
$$

${ }^{*}$ Department of Mathematical Sciences, Rensselaer Polytechnic Institute. Email: brownc12@rpi.edu

${ }^{\dagger}$ Department of Mathematics, University of Wisconsin-Madison. Email: aldickerson@wisc.edu

${ }^{\ddagger}$ School of Mathematics, University of Minnesota. Email: gfaye@umn.edu

${ }^{\S}$ School of Mathematics, University of Minnesota. Email: scheel@umn.edu 
we obtain through rescaling $v_{j}^{\prime}=\varepsilon^{2} \dot{u}_{j}, v_{j}=\varepsilon u_{j}$, the form (1.1). Similar scalings can be used to show how pitchfork and cusp bifurcations arise from the unfolding of singularities in the intracellular dynamics in the presence of weak spatial coupling. We shall see later that, in some sense, weak spatial coupling is necessary for a "local" description near threshold; see Remark 3.3.

While we do not claim that one could validate (1.1) as a somehow universal description of instabilities in infinite systems, those equations have natural appeal beyond being leading-order descriptions of elementary bifurcations in the intracellular dynamics. We think of these examples as the (arguably) simplest systems where one can study spatial competition between stable and unstable states (fold) and between two stable states (cusp). The pitchfork example can be thought of as a caricature of the possibly more relevant Hopf bifurcation.

One may interpret the system (1.1) as the discretization of the simple advection equation

$$
\partial_{t} u=f(u)+\operatorname{sgn}(\alpha) \partial_{x} u .
$$

However, this interpretation is only appropriate for $\alpha<0,|\alpha| \gg 1$. For $\alpha>0$, the discretization is ill-posed and generates numerical oscillations, which will manifest as "flip flops", in our network. For $|\alpha| \ll 1$, one expects to see discretization effects, with a plethora of solutions. In fact, one readily notices that for $\alpha=0$, all equations decouple, and the set of stationary solutions is isomorphic to $\left\{f^{-1}(0)\right\}^{\mathbb{Z}}$.

Our main motivation comes from recent work in [6] and, more specifically, [9]. In these articles, bifurcations in coupled cell networks are studied systematically, exploiting normal form transformations in the presence of specific network structures to give predictions for dynamics near instabilities. One of the most striking observations is the fact that bifurcations lead to solutions exhibiting a rapid growth in amplitude as a function of cell index. For instance, Hopf bifurcations lead to amplitudes $\mu^{\frac{1}{2} \frac{1}{3^{j-1}}}$ at cell position $j$, where $\mu$ represents a principal bifurcation parameter. The semi-infinite feed-forward network therefore acts as an effective amplifier of the input dynamics in cell $j=0$ (which is of course independent of the dynamics in cells $j>0$ ). The particular dynamics at cell $j=0$ are motivated by the presence of "symmetries", which require dynamics in all cells to be identical in the sense that the same number of inputs is received. In this sense, the input $u_{j-1}$ is replaced by $u_{j}$ for cell $j=0$, leading to a cancellation and vanishing of the coupling term $\alpha\left(u_{j}-u_{j-1}\right)$.

The rapid growth of equilibrium amplitudes with $j$ also points to limitations of a local bifurcation analysis. In particular, one expects that the expansions of these quantities are only valid for

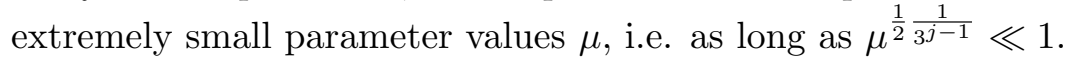

Our main objective is to elucidate this phenomenon and provide a conceptual analysis beyond the limitations of a local analysis for specific models. We therefore rely on the very simple models described above, which we hope capture many of the key features from the considerably more general setup in [9]. Our first main observation is that the instability studied in [9] can be understood, at least in these prototypical examples, as a transition from a convective to an absolute instability $[1,8,12]$. In the "stable" regime, perturbations grow in amplitude but are transported along the lattice so that they decay at any fixed initial location $j$. The speed of transport $c$ decreases as the parameter approaches a critical value, at which point stationary solutions bifurcate. The instability can therefore be understood as the transition from a receding invasion front to a stationary interface. In other contexts, the emerging stationary interfaces that typically connect the unstable state to a large, finite-amplitude state at the end of the lattice $j \rightarrow \infty$, are often referred to as nonlinear global modes $[3,5]$. 
We analyze this transition in detail and uncover how the receding invasion front picks up an increasingly rapid exponential decay as the speed converges to zero. This steepening of the front can be understood as a precursor to the existence of stationary profiles exhibiting a rapid growth in amplitude with $j$. Intuitively, exponential, temporal growth generated by the instability becomes sufficiently strong to compensate for the effective transport towards increasing $j$ and would, in a non-feed-forward situation, lead to transport towards $j=-\infty$, that is, negative speeds would emerge. Clearly, such transport is prohibited by our network structure, where cells of index $j_{*}$ simply ignore cell dynamics at $j>j_{*}$. As a result, the invasion front remains stationary despite increasing strength of the instability, a state which we refer to as a frustrated front. Mathematically, we can calculate the steepening of the spatial decay as the speed approaches zero, as it is mirrored by the disappearance of a double root at infinity in the complex plane. We interpret this steepening as a precursor to the rapid spatial growth of frustrated fronts, found (in finite systems, in a bifurcation setting) in $[6,9]$.

Beyond these stable-unstable interfaces responsible for transitions from convective to absolute instability, we study existence and stability of more general interfaces between stable states in the system. We choose to focus on interfaces between the most prevalent (and from a numerical discretization point of view most dangerous) of these stable states, namely, spatially homogeneous, $j$-independent, states and period-two states; see Remark 2.5. Our main result concerning those types of interfaces is a simple criterion that gives stability information and existence of interfaces based on readily computable properties of an interval map for spatial dynamics. We also illustrate the effectiveness of these criteria in a systematic numerical exploration of parameter space.

The remainder of this paper is organized as follows. Section 2 contains our main general results on stability of period-two equilibria and existence of interfaces, independent of the specific shape of $f$. Section 3 contains the analysis of fold and pitchfork, including spreading speed calculations. Section 4 treats the more complex case of the cusp.

\section{Coherent Structures and Interval Maps}

The core of this section is the relationship between the existence of stationary interfaces, i.e. temporally stable network configurations, and dynamics of an interval map produced from (1.1). Throughout the remainder of this section, we will ignore the first node in our network (though we will later comment on the effects of this truncation), and extend our analysis to a bi-infinite chain,

$$
\dot{u}_{j}=f\left(u_{j}\right)+\alpha\left(u_{j}-u_{j-1}\right), \forall j \in \mathbb{Z}, \alpha \neq 0 .
$$

\subsection{Equilibria and Stability}

Seeking a method to compute the values of the temporal equilibria of each subsequent cell in our system, we set $\dot{u}_{j}=0$ in (2.1), producing the one-term backward recursion

$$
u_{j-1}^{*}=g\left(u_{j}^{*}\right):=u_{j}^{*}+\frac{f\left(u_{j}^{*}\right)}{\alpha} .
$$

Throughout this paper, we will consistently differentiate between the temporal dynamics of our system, related to (2.1), and the spatial (in $j$ ) dynamics of our system corresponding to the sequence $\left(u_{j}^{*}\right)_{j \in \mathbb{Z}}$, the orbit under $g$ of our temporal steady states. 
Lemma 2.1. Spatially homogeneous ( $j$-independent) equilibria $u_{j}^{*} \equiv \bar{u}, j \in \mathbb{Z}$ of (2.1) are given precisely by the zeros of $f$. These equilibria are linearly asymptotically stable for our temporal dynamics precisely when they are linearly unstable for the spatial dynamics (when $\left|g^{\prime}(\bar{u})\right|>1$ ) and when $\alpha g^{\prime}(\bar{u})<0$.

Proof. It suffices to show that the spectrum of the bi-infinite matrix obtained by linearizing (2.1) is strictly contained in the left hand side of the complex plane precisely when $\left|g^{\prime}(\bar{u})\right|>1$ and $\alpha g^{\prime}(\bar{u})<0$. To this end, we employ a Fourier transform, $u_{j}(t)=\hat{u}_{\nu}(t) e^{i j \nu}$ to change our linearization

$$
\dot{u}_{j}=f^{\prime}(\bar{u}) u_{j}+\alpha\left(u_{j}-u_{j-1}\right), \quad j \in \mathbb{Z},
$$

into

$$
\dot{\hat{u}}_{\nu} e^{i j \nu}=f^{\prime}(\bar{u}) \hat{u}_{\nu} e^{i j \nu}+\alpha\left(\hat{u}_{\nu} e^{i j \nu}-\hat{u}_{\nu} e^{i j \nu} e^{-i \nu}\right), \quad j \in \mathbb{Z} .
$$

Upon substitution of the ansatz $\hat{u}_{\nu}(t)=e^{\lambda t}$ into (2.3), we find the dispersion relation

$$
\lambda(\nu)=f^{\prime}(\bar{u})+\alpha\left(1-e^{-i \nu}\right)
$$

We see that for $\alpha>0$ we will have $\operatorname{Re}(\lambda(\nu))<0$ precisely when $f^{\prime}(\bar{u})+2 \alpha<0$, while for $\alpha<0$, we will require $f^{\prime}(\bar{u})<0$. Recalling $(2.2)$, we see that

$$
f^{\prime}(\bar{u})=\alpha\left(g^{\prime}(\bar{u})-1\right) .
$$

Thus, we see that for $\alpha>0, f^{\prime}(\bar{u})+2 \alpha<0$ precisely when $g^{\prime}(\bar{u})<-1$, while for $\alpha<0, f^{\prime}(\bar{u})<0$ precisely when $g^{\prime}(\bar{u})>1$. Then noting that our condition $\alpha g^{\prime}(\bar{u})<0$ effectively forces $g^{\prime}(\bar{u})$ and $\alpha$ to be of opposite sign, we see that for $\alpha>0, g^{\prime}(\bar{u})<0$, while for $\alpha<0, g^{\prime}(\bar{u})>0$. This, together with the conditions discussed in the previous paragraph, ensures that we will have $\operatorname{Re}(\lambda)<0$ precisely when $\left|g^{\prime}(\bar{u})\right|>1$ and $\alpha g^{\prime}(\bar{u})<0$, from which the result follows.

We now turn our attention to describing period-two solutions of (2.2), which we will refer to as "flip flops".

To compute explicitly these period two equilibria, we reduce (2.1) to the system

$$
\begin{aligned}
& \dot{u}_{0}=f\left(u_{0}\right)+\alpha\left(u_{0}-u_{1}\right), \\
& \dot{u}_{1}=f\left(u_{1}\right)+\alpha\left(u_{1}-u_{0}\right) .
\end{aligned}
$$

Equilibrium solutions of this period-two chain, $u_{0}=u_{\text {flip }}, u_{1}=u_{\text {flop }}$ will themselves be equilibria on our bi-infinite lattice, that is, $u_{2 j}=u_{\text {flip }}, u_{2 j+1}=u_{\text {flop }}, j \in \mathbb{Z}$ will constitute an equilibrium solution of (2.1).

Lemma 2.2. A non homogeneous, period two equilibrium of (2.1), $u_{0}=u_{\text {flip }}, u_{1}=u_{\text {flop }}$, is linearly stable in our temporal dynamics precisely when $\left|G^{\prime}\left(u_{f}\right)\right|>1$ and $\alpha g^{\prime}\left(u_{f}\right)<0$ for both $u_{f}=u_{\text {flip }}$ and $u_{f}=u_{\text {flop }}$, where $G=g \circ g$.

\section{Proof.}

From our definition of $G$, we note that

$$
G^{\prime}\left(u_{\text {flip }}\right)=g^{\prime}\left(u_{\text {flip }}\right) g^{\prime}\left(g\left(u_{\text {flip }}\right)\right)=g^{\prime}\left(u_{\text {flip }}\right) g^{\prime}\left(u_{\text {flop }}\right)=g^{\prime}\left(g\left(u_{\text {flop }}\right)\right) g^{\prime}\left(u_{\text {flop }}\right)=G^{\prime}\left(u_{\text {flop }}\right) .
$$


So, we will have both $\left|G^{\prime}\left(u_{\text {flip }}\right)\right|>1$ and $\left|G^{\prime}\left(u_{\text {flop }}\right)\right|>1$ precisely when $\left|g^{\prime}\left(u_{\text {flip }}\right) g^{\prime}\left(u_{\text {flop }}\right)\right|>1$.

To begin, we set $u_{2 j}=v_{j}, u_{2 j+1}=w_{j}$, and rewrite the linearization of our system (2.1) about our equilibrium $\left(u_{\text {flip }}, u_{\text {flop }}\right)$,

$$
\dot{v}_{j}=f^{\prime}\left(u_{\mathrm{flip}}\right) v_{j}+\alpha\left(v_{j}-w_{j-1}\right) \text { and } \dot{w}_{j}=f^{\prime}\left(u_{\mathrm{flop}}\right) w_{j}+\alpha\left(w_{j}-v_{j}\right) .
$$

Substituting $v_{j}(t)=\hat{v}_{\nu}(t) e^{-i j \nu}, w_{j}(t)=\hat{w}_{\nu}(t) e^{-i j \nu}$ into the above equations we obtain the Jacobian matrix

$$
J(\nu)=\left(\begin{array}{cc}
f^{\prime}\left(u_{\text {flop }}\right)+\alpha & -\alpha e^{-i \nu} \\
-\alpha & f^{\prime}\left(u_{\text {flip }}\right)+\alpha
\end{array}\right)
$$

where $\nu \in[0,2 \pi]$. We note that the trace of this matrix is independent of $\nu$ and real.

- We first assume temporal stability of our equilibrium. Thus we must have that the real part of the eigenvalues associated with $J(\nu)$ are strictly negative for all $\nu \in[0,2 \pi]$. In particular, at $\nu=0$, we must have both $\operatorname{det}(J(0))>0$, and $\operatorname{tr}(J)<0$. Noting that we may rewrite $\operatorname{det}(J(0))$ as

$$
\operatorname{det}(J(0))=\alpha^{2}\left(\left(\frac{f^{\prime}\left(u_{\mathrm{flip}}\right)}{\alpha}+1\right)\left(\frac{f^{\prime}\left(u_{\mathrm{flop}}\right)}{\alpha}+1\right)-1\right) .
$$

We see that by using (2.4), we may rewrite our condition $\operatorname{det}(J(0))>0$

$$
\operatorname{det}(J(0))=g^{\prime}\left(u_{\text {flip }}\right) g^{\prime}\left(u_{\text {flop }}\right)=G^{\prime}\left(u_{\text {flip }}\right)>1,
$$

and so $\left|G^{\prime}\left(u_{\text {flip }}\right)\right|>1$.

Then, noting that we may rewrite $\operatorname{tr}(J(0))<0$ as

$$
\left(\alpha g^{\prime}\left(u_{\text {flip }}\right)\right)+\left(\alpha g^{\prime}\left(u_{\text {flop }}\right)\right)<0
$$

and that $\alpha g^{\prime}\left(u_{\text {flip }}\right) \alpha g^{\prime}\left(u_{\text {flop }}\right)>0$, we immediately see that both $\alpha g^{\prime}\left(u_{\text {flip }}\right)$ and $\alpha g^{\prime}\left(u_{\text {flop }}\right)$ are negative. Thus, $\operatorname{tr}(J)<0$.

- Now reversing directions, and keeping in mind that the assumptions $\alpha g^{\prime}\left(u_{f}\right)<0$ and $\left|G^{\prime}\left(u_{f}\right)\right|>$ 1 for $u_{f}=u_{\text {flip }}, u_{f}=u_{\text {flop }}$ are equivalent to the conditions $\operatorname{tr}(J)<0$ and $\operatorname{det}(J(0))>0$, we will proceed to show temporal stability of our solution by showing that for all $\nu \in[0,2 \pi]$,

$$
\operatorname{Re}\left(\lambda_{ \pm}(\nu)\right)=\frac{\operatorname{tr}(J)}{2} \pm \operatorname{Re}(\sqrt{D(\nu)})<0
$$

where

$$
D(\nu)=\frac{\operatorname{tr}^{2}}{4}-\operatorname{det}(J(\nu))=\left(\frac{f^{\prime}\left(u_{\mathrm{flip}}\right)-f^{\prime}\left(u_{\mathrm{flop}}\right)}{2}\right)^{2}+\alpha^{2} e^{-i \nu}
$$

denotes the discriminant of $J(\nu)$. As we are already have that $\left.\operatorname{Re}\left(\lambda_{ \pm}(0)\right)=\lambda_{ \pm}(0)\right)<0$, it will suffice to show that

$$
\operatorname{Re}(\sqrt{D(\nu)})<\sqrt{D(0)}, \quad \forall \nu \in[0,2 \pi] .
$$

Writing $D(\nu)=A+B e^{-i \nu}, A, B>0$, we begin by rewriting (2.10) as

$$
\sqrt{A+B e^{i \nu}}+\sqrt{A+B e^{-i \nu}}<2 \sqrt{A+B}, \quad \forall \nu \in[0,2 \pi] .
$$


Taking without loss of generality $A=1$, and squaring both sides of (2.11), we find the positive, real, relation

$$
2+2 B \cos (\nu)+2 \sqrt{1+B^{2}+2 B \cos (\nu)}<4+4 B
$$

which is equivalent to the obviously valid relation

$$
1+B^{2}+2 B \cos (\nu)<\frac{(2+2 B(2-\cos (\nu)))^{2}}{4}=1+B^{2}(2-\cos (\nu))^{2}+2 B(2-\cos (\nu)) .
$$

Thus for all $\nu \in[0,2 \pi]$ we have $\operatorname{Re}(\sqrt{D(\nu)})<\sqrt{D(0)}$, and so $\operatorname{Re}\left(\lambda_{ \pm}(\nu)\right)<0$. Thus, our equilibrium is temporally stable.

Remark 2.3. In later sections, where we discuss the temporal and spatial stability of particular equilibria for specific $f$, it will suffice to consider only the case $\nu=0$, as we have shown that stability in this case will imply stability $\forall \nu \in[0,2 \pi]$.

Corollary 2.4. Period-two equilibria are temporally stable precisely when they are stable in a period-two chain, that is, when $j \in \mathbb{Z} /(2 \mathbb{Z})$.

Remark 2.5. While higher period solutions to (2.1) may exist, we will examine only homogeneous and flip flop solutions. When $\alpha=0$, we see that the set of equilibria of (2.1) is isomorphic to $\left\{f^{-1}(0)\right\}^{\mathbb{Z}}$. We will not attempt to describe how this plethora of solutions evolves as $|\alpha|$ is increased. By Sharkovskii's theorem [13], existence of periodic orbits other than period 1 or period 2 imply the existence of periodic orbits of period 1 and 2. In other words, period 1 and period 2 orbits occupy the largest parameter regime. If interpreting periodic orbits with period larger than one as numerically dangerous oscillations, one therefore concludes that period 2 orbits are most prevalent, hence most dangerous.

\section{$2.2 \quad$ Stationary Interfaces}

We now define our first coherent network structure in terms of heteroclinic connections, which we will refer to as a stationary profile, as follows. A heteroclinic connection between two equilibria is a spatial trajectory, $\left(u_{j}\right)_{j \in \mathbb{Z}}$, joining $u_{+}$at $j=\infty$, and $u_{-}$at $j=-\infty$. We will also consider heteroclinic connections to flip flop equilibria, i.e. $\left|u_{2 j}-u_{\text {flip }}\right|+\left|u_{2 j+1}-u_{\text {flop }}\right| \rightarrow 0$ as $j \rightarrow \infty$. We say that a stationary profile exists between two states, $A$ at $j=-\infty$ and $B$ at $j=\infty$, if and only if such a heteroclinic connection exists between these two states. Throughout this paper, we will use the notation $A \rightarrow B$, to denote the existence of a stationary profile between $A$ and $B$. Further, the notation $F F \rightarrow B$, will denote a stationary profile whose left hand side (at $j=-\infty)$ is a flip flop equilibrium.

Lemma 2.6. Let the state $\bar{u}$ at $j=-\infty$ be stable for the temporal dynamics of our system. Then, a heteroclinic cannot exist if there does not exist $u_{p} \neq \bar{u}$ such that $g\left(u_{p}\right)=\bar{u}$. Similarly, heteroclinic connections to flip flops, $\bar{u}_{\text {flip }}, \bar{u}_{\text {flop }}$ as $j=-\infty$ cannot exist when there exists no $u_{p} \notin\left\{\bar{u}_{\text {flip }}, \bar{u}_{\text {flop }}\right\}$, such that $g\left(u_{p}\right)=\bar{u}_{\text {flip }}$ or $g\left(u_{p}\right)=\bar{u}_{\text {flop }}$.

Proof. Let $\bar{u}$ denote a temporally stable equilibrium solution at $j=-\infty$, whose only preimage under $g$ is $\bar{u}$, but with $u_{j} \rightarrow u_{p}$ as $j \rightarrow \infty$. By Lemma 2.1, this cannot happen, as a temporally stable state must be unstable for our spatial, $g$, dynamics, and so $\bar{u}$ is a repeller for our map $g$. 
Remark 2.7. There do not exist heteroclinic connections to a temporally unstable state at $j=\infty$, as these states are stable for our map g by Lemma 2.1, and therefore there exist no trajectories converging to them in backward spatial iteration.

Lemma 2.8. In semi-infinite networks, i.e., when $0 \leq j<N, N=\infty$, the conditions of Lemma 2.6 may be extended to include temporally unstable states at $j=0$.

Proof. Let the state $\bar{u}_{1}$ be a temporally unstable solution at $j=0$, whose only preimage under $g$ is $\bar{u}_{1}$. Let $\bar{u}_{2}$ denote an equilibrium solution at $j=\infty, \bar{u}_{1} \neq \bar{u}_{2}$. If a heteroclinic connection existed between the two states in question, it would follow that there exists $u_{p} \neq \bar{u}_{1}$ such that, $g^{-1}\left(u_{p}\right) \neq \bar{u}_{1}$, which is clearly a contradiction.

Remark 2.9. In semi-infinite networks, there exists a heteroclinic connection between the state $A$ at $j=0$ and $B$ at $j=\infty$ if and only if there exists a preimage $u_{p}$ such that $g\left(u_{p}\right)=A$ and the subsequent preimages of $u_{p}, g^{-j}\left(u_{p}\right), j \in \mathbb{Z}^{+}$satisfy $g^{-j}\left(u_{p}\right) \rightarrow B$ as $j \rightarrow \infty$.

Remark 2.10. If there exists a stationary profile in a semi-infinite network, then there exists an analogous stationary profile in an infinite network.

Remark 2.11. Lemma 2.6 gives a quite simple necessary condition for the existence of a heteroclinic. One can give sufficient conditions in various flavors. Roughly speaking, the nontrivial preimage of the state at $j=-\infty$ needs to be contained in the unstable manifold of the state at $+\infty$. For fixed points at $j=+\infty$, the unstable manifold contains an open neighborhood of that fixed point, bounded by different fixed points or period-2 orbits of $g$. Similarly, the unstable manifold of period-2 orbits is bounded by period-2 or period-4 orbits. In the examples, below, we found by visual inspection that the necessary criterion, Lemma 2.6 always implied this sufficient criterion, although an algebraic verification could possibly be quite tedious.

\subsection{Moving Interfaces: Propagation into Unstable States}

Taking motivation from the original bifurcation studies of [9] carried out in finite networks, we focus on describing possible bifurcations on our infinite lattice, corresponding to the transition between convective and absolute spreading of instabilities through our network.

To find these bifurcation points, we will attempt to describe the speed at which instabilities propagate from cell to cell. In many cases, commonly referred to as the case of pulled fronts, these speeds can be derived from a purely linear analysis, that we will outline now; see for instance [11, $\S 2.4]$. We start by linearizing (2.1) at a state $\bar{u}, f(\bar{u})=0$,

$$
\dot{u}_{j}=f^{\prime}(\bar{u}) u_{j}+\alpha\left(u_{j}-u_{j-1}\right), \quad j \in \mathbb{Z} .
$$

The initial-value problem can readily be solved using Fourier transformation, setting

$$
u_{j}=\int_{\sigma=0}^{2 \pi} e^{\lambda t} e^{i j \sigma} \hat{u}(\sigma) \mathrm{d} \sigma
$$

where $\lambda, \sigma$ are roots to the dispersion relation

$$
d(\lambda, i \sigma)=\lambda-f^{\prime}(\bar{u})-\alpha\left(1-e^{-i \sigma}\right)=0 .
$$

We are interested in pointwise decay of the solution in a comoving frame, given compactly (in $j$ ) supported initial conditions. In other words, we are interested in the behavior of $u_{j-c t}$ as $t \rightarrow \infty$, 
where of course the index is only meaningful for $c t \in \mathbb{Z}$, but can be formally evaluated in (2.12) for any $c, t$. For any fixed $c$, one can now deform the integral in (2.12) into the complex $\sigma$-plane, exploiting the fact that $\hat{u}(\sigma)$ is an entire function as a result of $u_{j}$ being compactly supported. Attempting to minimize the maximum of $\operatorname{Re} \lambda(\sigma)$ along this path, one arrives at double roots of $d$,

$$
d(\lambda-c \nu, \nu)=0, \quad \partial_{\nu} d(\lambda-c \nu, \nu)=0,
$$

where $\nu=\mathrm{i} \sigma \in \mathbb{C}^{1}$.

Double roots give exponential growth or decay rates in the frame $j=c t$. Spreading speeds are values of $c$ where those rates are neutral, $\operatorname{Re} \lambda=0$. We therefore look for solutions to

$$
d(i \omega-c \nu, \nu)=0, \quad \partial_{\nu} d(i \omega-c \nu, \nu)=0,
$$

with $\omega, c \in \mathbb{R}, \nu \in \mathbb{C}$. In the simplest case, which turns out to be relevant here for $\alpha<0, \omega=0$ and $\nu \in \mathbb{R}$, so that we need to solve two real equations,

$$
d(-c \nu, \nu)=0, \quad \partial_{\nu} d(-c \nu, \nu)=0
$$

Remark 2.12. The double root analysis was first used in the study of plasma instabilities; see [1] and references therein. Double roots with positive real part of $\lambda$ correspond to absolute instabilities (in a given, fixed coordinate frame). Double roots with $\operatorname{Re} \lambda<0$ correspond to convective (in)stability. Spreading speeds correspond to coordinate frames of marginal absolute stability; varying $c$ induces a transition from absolute to convective instability.

In our specific case, the double root criterion (2.15) gives

$$
f^{\prime}(\bar{u})+\alpha\left(1-e^{-\nu}\right)+c \nu=0, \quad \alpha e^{-\nu}+c=0 .
$$

Substituting $\nu$ from the second equation of (2.16) into the first equation gives

$$
\nu=\log \left(\frac{c}{\alpha}\right), \quad f^{\prime}(\bar{u})+\alpha-c+c \log \left(\frac{c}{\alpha}\right)=0 .
$$

The solution can be expressed explicitly in terms of the Lambert function $W$, defined as the solution to

$$
W(x) e^{W(x)}=x
$$

as

$$
c=\frac{f^{\prime}(\bar{u})+\alpha}{W\left(-\frac{f^{\prime}(\bar{u})+\alpha}{e \alpha}\right)} .
$$

Since the Lambert function has two real branches only, equations (2.15) have at most two solutions, $c_{1}, c_{2}$. More precisely, there exist two branches $W_{0}(x)>W_{-1}(x)$ for $x \in\left(-e^{-1}, 0\right]$ and only one branch $W_{0}$ for $x>0$; see for instance [4]. In our case, this gives (assuming $\alpha>0$ ),

- two wave speeds $c_{0}, c_{-1}$ for $f^{\prime} \in(0,-\alpha)$ (strong coupling), associated with $W_{0}, W_{-1}$, respectively;

- one wave speed $c_{0}$ for $f^{\prime}>-\alpha$ (weak coupling), associated with $W_{0}$.

\footnotetext{
${ }^{1}$ In general, the double-root condition needs to be supplemented with a pinching condition, which turns out to be satisfied automatically in our case here; we refer to $[1,8]$ for a more extensive discussion
} 
We will see how the precise moment of transition from convective to absolute instability coincides with the moment at which one of our speeds vanishes.

Remark 2.13. In a network with initial condition $u_{j}=A$ for $j \leq j^{*}, A$ stable and $u_{j}=B$ for $j>$ $j^{*}, B$ unstable, the speed at which the state $A$ "invades" $B$ will be given by $c=\max \left\{c_{1}, c_{2}\right\}$; see [11] for details.

Remark 2.14. Due to the properties of our network coupling, negative wave speeds will not be possible. This means that a stable state cannot invade an unstable state to the left, leading to the presence of what we will call "Frustrated Invasion Fronts", whose propagation is blocked by our network structure.

Remark 2.15. Beyond our linear analysis, one may consider nonlinear front solutions. There typically exists a one-parameter family of such fronts and the steepest front is selected by compactly supported initial conditions. Existence of such fronts leads to delay equations rather than the map $g$ and has been studied with slightly different objectives in [7]. Similarly, information about traveling fronts that connect stable equilibria also leads to delay equations and is beyond the scope of this article.

The most striking phenomenon is the vanishing of the second wave speed $c_{-1}$ at $f^{\prime}=-\alpha$, leading to the frustrated stationary front. Considering $\alpha<0$ as a parameter and fixing $f^{\prime}$, we can investigate the limit of vanishing wave speeds, using asymptotics of the Lambert function.

$$
\begin{aligned}
c_{-1}(\alpha) & \sim-\frac{\Delta \alpha}{\log \Delta \alpha}, \text { as } \Delta \alpha=-\alpha-f^{\prime}(\bar{u}) \rightarrow 0^{+}, \\
c_{0}(\alpha) & \sim-\frac{2}{\log (-\alpha)}, \text { as } \alpha \rightarrow 0^{-} .
\end{aligned}
$$

Note that, in both cases, $\nu=\log c-\log (\alpha)$ converges to $-\infty$ as $c \rightarrow 0$, reflecting a steepening of the invasion front as it approaches the frustrated regime.

\section{Fold and Pitchfork Bifurcations}

In this section, we examine the two specific nonlinearities, $f(u)=1-u^{2}$, and $f(u)=u\left(1-u^{2}\right)$, associated with the normal forms of the saddle node and pitchfork bifurcations respectively. We will later see that by understanding the dynamics of this system, we gain valuable insight into more complicated dynamics such as the nonlinearity associated with the normal forms of the cusp bifurcation.

\subsection{Fold Bifurcation}

\subsubsection{Temporal Stability of Steady States}

Letting $f(u)=1-u^{2}$, we begin by characterizing the conditions on $\alpha$ which will permit the existence and temporal stability of various period-two (i.e. homogeneous and flip flop) equilibria of (2.1).

Lemma 3.1. There exist precisely two homogeneous equilibria of our system corresponding to the states -1 and 1 . For $|\alpha|<1$, there also exists one flip flop equilibrium given by $u_{2 j} \equiv \alpha+\sqrt{1-\alpha^{2}}$ and $u_{2 j+1} \equiv \alpha-\sqrt{1-\alpha^{2}}$, for all $j \in \mathbb{Z}$. Furthermore, we find that the states -1 and flip flop are always temporally unstable, while 1 is temporally stable provided $\alpha<1$. 
Proof. Our desired result follows by a simple application of Remark 2.3 and Corollary 2.4. To verify, for instance, the stability of our flip flop equilibrium, we inspect the Jacobian of our 2-cell system

$$
J(0)=\left(\begin{array}{cc}
f^{\prime}\left(u_{0}\right)+\alpha & -\alpha \\
-\alpha & f^{\prime}\left(u_{1}\right)+\alpha
\end{array}\right),
$$

from which we derive the eigenvalues associated with our flip flop equilibrium,

$$
\lambda_{\mathrm{ff}_{ \pm}}=-\alpha \pm \sqrt{4-3 \alpha^{2}}
$$

Noting that for $|\alpha|<1, \lambda_{\mathrm{ff}_{+}}>0$, temporal instability follows.

\subsubsection{Stationary Interfaces}

Considering both semi-infinite and bi-infinite length chains, we now discuss the existence of stationary interfaces in our network. We know from Remark 2.7 that we can expect to observe at most two stationary interfaces in our system, each of form $-1 \rightarrow 1$ and $F F \rightarrow 1$ respectively. Further, since 1 is stable only when $\alpha<1$, and since flip flops only exist for $|\alpha|<1$, we already have upper bounds on the region of existence for these structures.

Lemma 3.2. We find for semi-infinite networks that stationary profiles of form $-1 \rightarrow 1$ exist precisely for $-2<\alpha<1$, while for bi-infinite networks, such a profile exists for $\alpha<1$. Stationary profiles of form $F F \rightarrow 1$ exist for $|\alpha|<1$ in both semi-infinite and bi-infinite networks.

Proof. Here we use Remarks 2.9 and 2.10 to derive conditions on $\alpha$ which will permit the existence of various stationary profiles.

To determine the bounds of existence for stationary profiles of form $-1 \rightarrow 1$, we examine case by case the preimages of -1 under $g$ for various $\alpha$.

- $0<\alpha<1$ : With our just mentioned remarks in mind, we note that in this parameter regime, there exists $u_{p} \neq-1$ such that $g\left(u_{p}\right)=-1$. Further, subsequent preimages of $u_{p}$ converge to 1 as $j \rightarrow \infty$, and so we conclude for both semi-infinite and bi-infinite networks that a $-1 \rightarrow 1$ stationary profile exists.

- $-2<\alpha<0$ : Here, we find precisely the same behavior as in the previous region, and thus arrive at the same conclusion. An illustrative example of such a heteroclinic connection is given in Figure 1.

- $\alpha=-2$ : We note that, as the minimum of $g$ now occurs at $u_{\text {min }}=-1$, we no longer have our desired $u_{p} \neq-1$ such that $g\left(u_{p}\right)=-1$. Thus, we conclude that for a semi-infinite network, there exists no heteroclinic connection between -1 and 1 , and so no such stationary profile exists. Interestingly, we still have the existence of such a connection, and thus a stationary profile, in our bi-infinite network (one can imagine such a heteroclinic by observing Figure 1).

- $\alpha<-2$ : In our final case, we see no change in behavior from $\alpha=-2$ in our bi-infinite network. A heteroclinic connection between -1 and 1 still exists, and so we still have a $-1 \rightarrow 1$ stationary profile. In the semi-infinite case, -1 regains its preimage, $u_{p} \neq-1$, however, subsequent preimages of $u_{p}$ fail to exist under $g$, and so we have no stationary profile. 
We use similar methods to uncover the regions of existence for $F F \rightarrow 1$ stationary profiles for any $|\alpha|<1$. For example, in the parameter region $0<\alpha<1$, we note that there always exists $u_{p} \neq \alpha-\sqrt{1-\alpha^{2}}$ such that $g\left(u_{p}\right)=\alpha+\sqrt{1-\alpha^{2}}$. In fact, $u_{p}$ is given in closed form as $u_{p}=\sqrt{1-\alpha^{2}}$. Finally, as before, further subsequent preimages of $u_{p}$ converge to 1 as $j \rightarrow \infty$, and so we conclude for both semi-infinite and bi-infinite networks that a $F F \rightarrow 1$ stationary profile exists. The exact same argument works for $-1<\alpha<0$.

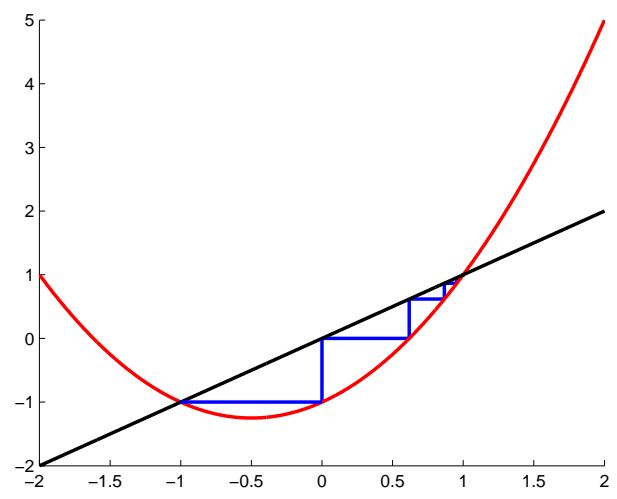

Figure 1: An example of 15 iterations under the map $g$ when $\alpha=-1$. As the point -1 has a preimage under $g$, we find a heteroclinic connection joining -1 to 1 at $j=\infty$, implying the existence of a $-1 \rightarrow 1$ stationary profile. The diagonal has been shown for illustrative purposes.

\subsubsection{Linear Wave Speeds}

Armed with a complete understanding of conditions on $\alpha$ which force the existence of a stationary front we now turn our attention to characterizing the speed at which invading fronts propagate through our network. Throughout we assume $\alpha \leq 0$, which gives real solutions to our double-root equation.

We use (2.18) with $\bar{u}=-1$ and $f^{\prime}(\bar{u})=2$, which gives

$$
c=\frac{2+\alpha}{W\left(-\frac{2+\alpha}{e \alpha}\right)},
$$

with two wave speeds for $\alpha<-2$ and one wave speed for $\alpha \in(-2,0)$.

Examining these explicit formulae for computing our wave speeds (2.18), we see that, as $\alpha \rightarrow-2$, $c_{-1} \rightarrow 0$, our network undergoes a bifurcation from a convective to absolute instability about -1 , corresponding to the bifurcations discussed by [9]. Of further interest is that this is the precise moment at which we gain the existence of a $-1 \rightarrow 1$ stationary profile, that is, as $\alpha \rightarrow-2$, we see an increasingly sharp restoring wave, which eventually degenerates into a stationary profile. A visual representation of this information may be found in Figure 2.

Remark 3.3. From the point of view of bifurcation theory, we saw that a simple rescaling as in (1.2) gives strong coupling unless the original coupling is assumed to be weak. As a consequence, we would not observe the transition that is of particular interest here. A different point of view is that in 

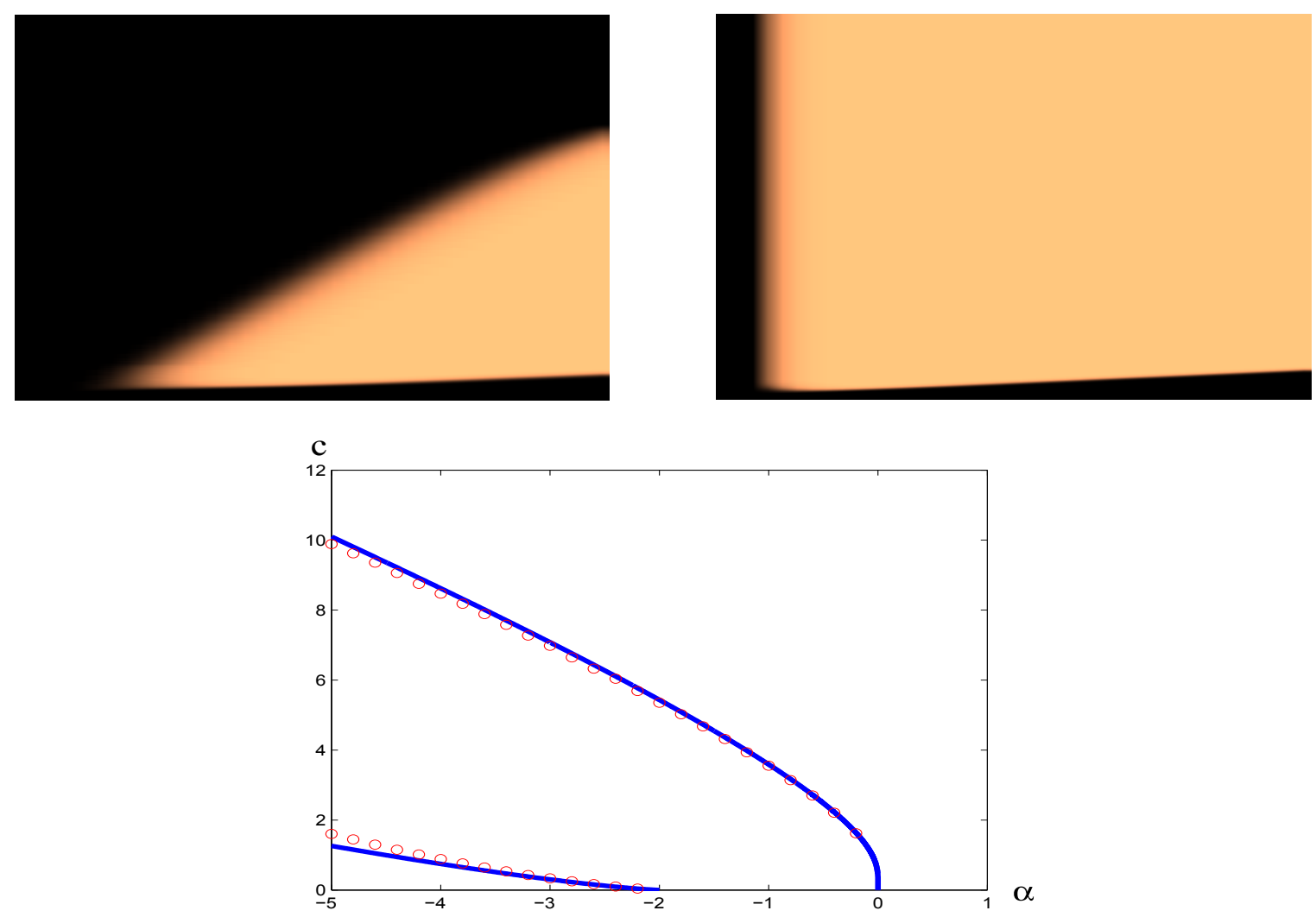

Figure 2: The top two pictures show numerical simulations of a feed-forward network with 40 cells (horizontally) over 300 time units (vertically). Black denotes the state 1 , while copper denotes -1 . The bottom picture shows such a simulation for $\alpha=-3$, at which point both $c_{-1}, c_{0} \neq 0$. Thus, we see a convecting instability. In contrast, the middle picture, a simulation with $\alpha=-1$, shows the propagation of an absolute instability, which remains indefinitely. The final figure shows the two analytically computed branches of (2.18), together with numerically computed, nonlinear wave speeds shown as dots.

non-symmetric systems, the onset of convective and absolute instabilities differ, so that, at the onset of absolute instabilities, the primary stable branch of equilibria that bifurcated at the onset of the convective instability has already reached a finite, non-small amplitude, and the transition cannot be captured in a universally valid amplitude expansion. The remedy is to consider cases where "asymmetry" is weak, pulling onset of convective and absolute instability together in parameter space, which in our case is accomplished by assuming weak coupling.

As a closing note, we could in principle apply the same techniques discussed throughout the two previous sections to find the various wave speeds in the case $\alpha>0$, or even for our unstable flip flop equilibria. Formulae for wave speeds are not explicit in both cases and one relies on numerical solutions of the double-root equation. We tested those solutions and found good agreement with direct simulations. Phenomena are however similar in spirit to the ones reported here.

\subsection{Pitchfork Bifurcation}

With our discussion of the fold nonlinearity now complete, we next examine $f(u)=u\left(1-u^{2}\right)$. Here, we will repeat a similar analysis to our previous section though we will restrict our analysis to semi-infinite networks. To begin, we will address constraints on $\alpha$ which permit the existence 
and stability of various equilibria and specific stationary profiles.

\subsubsection{Existence and Stability of Equilibria}

Using precisely the same methods as in the previous section, we seek to find conditions on $\alpha$ which will permit the existence and stability of certain stationary profiles. To this end, we must first categorize the various equilibria of our system and their temporal stability.

Lemma 3.4. There exist precisely three homogeneous equilibria, $-1,1$, and 0 . There also exists up to three flip flop equilibria, given by:

- $u_{\mathrm{flip}_{1}}=\sqrt{1+2 \alpha}, \quad u_{\mathrm{flop}_{1}}=-\sqrt{1+2 \alpha}$ for $\alpha \geq-\frac{1}{2}$;

- $u_{\mathrm{flip}_{2}}=\frac{1}{2} \sqrt{2+2 \alpha+2 \sqrt{1+2 \alpha-3 \alpha^{2}}}, \quad u_{\mathrm{flop}_{2}}=\frac{1}{2} \sqrt{2+2 \alpha-2 \sqrt{1+2 \alpha-3 \alpha^{2}}}$ for $-\frac{1}{3} \leq \alpha \leq 1$

- $u_{\mathrm{flip}_{3}}=-\frac{1}{2} \sqrt{2+2 \alpha-2 \sqrt{1+2 \alpha-3 \alpha^{2}}}, \quad u_{\mathrm{flop}_{3}}=-\frac{1}{2} \sqrt{2+2 \alpha+2 \sqrt{1+2 \alpha-3 \alpha^{2}}}$ for $-\frac{1}{3} \leq \alpha \leq 1$.

Further, the temporal stability of these equilibria is characterized as follows:

- \pm 1 are stable for $\alpha<1$;

- 0 is always unstable;

- $u_{\mathrm{flip}_{1}}, u_{\mathrm{flop}_{1}}$ is stable for $\alpha>-\frac{1}{3}$;

- $u_{\mathrm{flip}_{k}}, u_{\mathrm{flop}_{k}}$ is always unstable for $k=2,3$.

In Figure 3, we show the values of all flip flop equilibria (and homogeneous equilibria) as a function of $\alpha$, and summarize the regions in which they are stable. Note that the value $\alpha=-\frac{1}{3}$ is the location of the pitchfork bifurcation denoted by diamonds in Figure 3, which in turn corresponds to the loss of stability of $\left(u_{\mathrm{flip}_{1}}, u_{\mathrm{flop}_{1}}\right)$.

Proof. The proof is identical to the proof of Lemma 3.1, and is thus omitted.

\subsubsection{Stationary Interfaces}

Though the general structure of this section will be similar to that of the fold, a complete evaluation of every possible stationary profile and traveling front associated with this network would be too lengthy, and so we will avoid such an analysis. Instead, we will examine interfaces between $0 \rightarrow 1$ and $0 \rightarrow F F$, where for the duration of this section, we will always assume the state FF corresponds to our flip flop equilibrium $u_{\mathrm{flip}_{1}}, u_{\mathrm{flip}_{1}}$. Further, we also note that by understanding the behavior of a $0 \rightarrow 1$ interface, we simultaneously gain insight into interfaces of form $0 \rightarrow-1$ thanks to the reflection symmetry of our system about 0 .

Lemma 3.5. In a semi-infinite network, we find that 


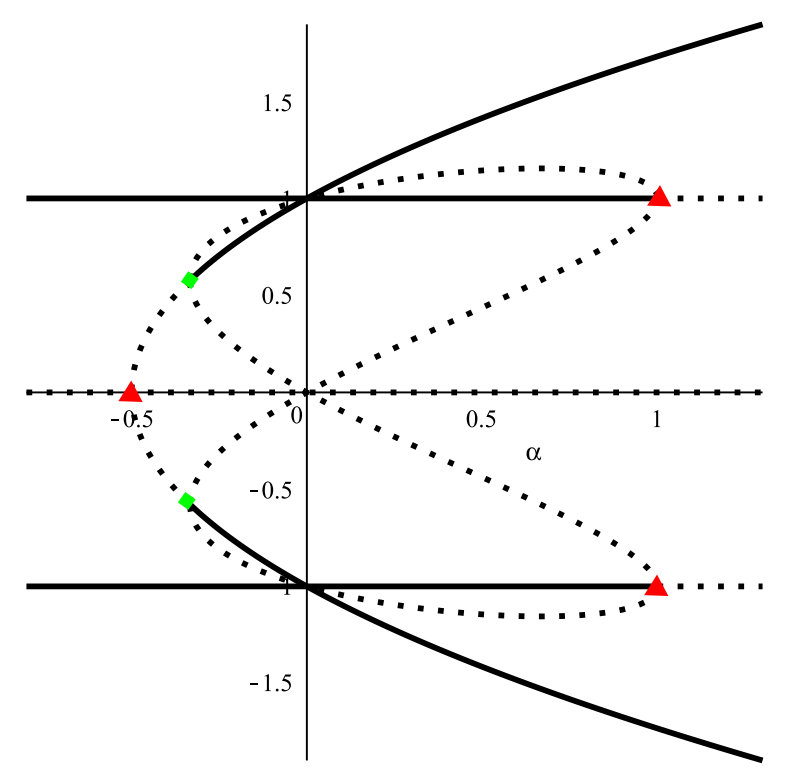

Figure 3: Bifurcation diagram in $\alpha$. Here, dotted lines represent temporally unstable equilibria, while solid represent temporally stable. The three horizontal lines correspond to the three homogeneous equilibria, and the remaining six curves correspond to the various flips and flops of Lemma 3.4. Specifically, the two outermost curves correspond to $u_{\mathrm{fli}_{1}}, u_{\mathrm{flip}_{1}}$. The four remaining curves give, going from highest to lowest, $u_{\mathrm{flip}_{2}}, u_{\mathrm{flop}_{2}}, u_{\mathrm{fli}_{3}}, u_{\mathrm{flop}_{3}}$. Triangles and diamonds represent the location of various pitchfork bifurcations, with the diamond pitchfork bifurcation being precisely the bifurcations referenced in Lemma 3.4.

- A stationary profile of form $0 \rightarrow F F$ exists provided $\alpha_{-}<\alpha$, where

$$
\alpha_{-}=\frac{4-6 \sqrt{3}}{23}
$$

- A stationary profile of form $0 \rightarrow 1$ exists provided $-1<\alpha<\alpha_{+}$, where

$$
\alpha_{+}=\frac{4+6 \sqrt{3}}{23} .
$$

Further, a $0 \rightarrow 1$ stationary profile exists precisely when a $0 \rightarrow-1$ profile exists.

An illustration of these results can be found in Figure 5.

Proof. Recalling Remark 2.9, a stationary profile of form $0 \rightarrow \bar{u}$ will exist precisely when there exists $u_{p} \neq 0$ such that $g\left(u_{p}\right)=0$, whose subsequent preimages under $g$ converge to $\bar{u}$.

- $\alpha<-1$ : Here, the point 0 ceases to have any preimage, $u_{p} \neq 0$, and so no stationary profile with state 0 at $j=0$ will exist.

- $-1<\alpha<\alpha_{-}$: Here we find two possible preimages of 0 which are equal in magnitude, $\pm u_{p}$. We find that the subsequent preimages under $g$ of $u_{p}$ exist and converge to 1 , while those of $-u_{p}$ converge to -1 . Thus, we conclude the existence of a $0 \rightarrow \pm 1$ stationary profile in this region. 


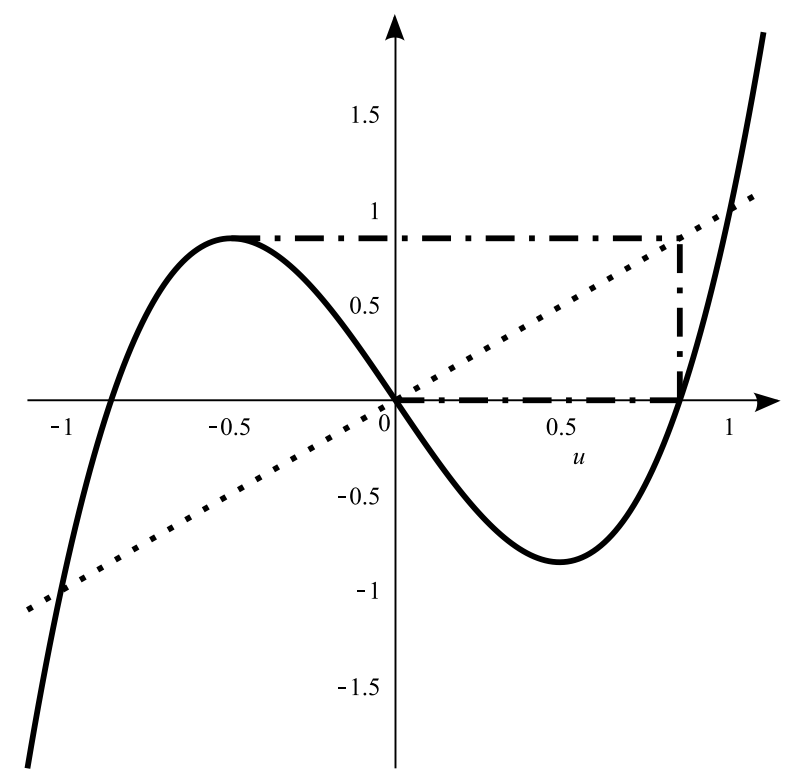

Figure 4: Preimages of 0 under $g$ when $\alpha \gtrsim \alpha_{-}$. Here, one can imagine four possible heteroclinic connections, corresponding to the four preimages of the points \pm 1 , which are themselves the two preimages of 0 .

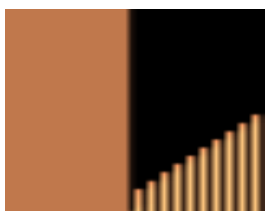

(a)

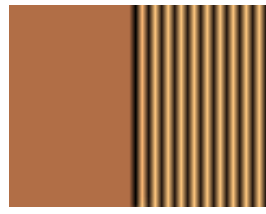

(b)

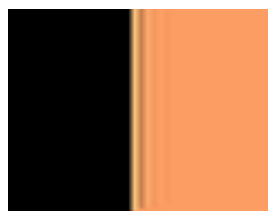

(c)

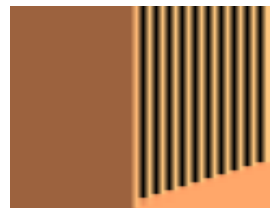

(d)

Figure 5: Numerical simulations of a semi-infinite pitchfork network using 350 time units (vertically) and 40 cells (horizontally). In (a), $\alpha \lesssim \alpha_{-}$, while in (b) $\alpha \gtrsim \alpha_{-}$and so we see the existence of a $0 \rightarrow F F$ stationary profile only in (b). Similarly, in (c), $\alpha \lesssim \alpha_{+}$, and in (d) $\alpha \gtrsim \alpha_{+}$, and so we see a $0 \rightarrow 1$ stationary profile in (c), but not in (d).

- $\alpha_{-}<\alpha<\alpha_{+}$: In this region, we find the existence of several possible heteroclinic connections to 0 at $j=0$. While heteroclinic connections joining 0 and \pm 1 are evident by graphical inspection (see Figure 4), the precise conditions which allow for a heteroclinic connection to flip flops are slightly more subtle. Denoting the two preimages of 0 on this interval as $\pm u_{p}$, we compute $\alpha_{-}$and $\alpha_{+}$by computing the precise moment at which there exists additional preimages of $\pm u_{p}$ in addition to those used in the heteroclinic connections to \pm 1 . Clearly, $\alpha_{-}$ is then found by the relation $g^{-1}\left(u_{p}\right)=u_{\max }$ where $u_{\max }$ denotes the local maximum of $g$ on $(-1,1)$, while $\alpha_{+}$is found using $g^{-1}\left(u_{p}\right)=u_{\min }$, where $u_{\min }$ denotes the local minimum of $g$ on the same interval. Alternatively, by inspecting the graph of the second iterate of $g$ one can recover the precise conditions which allow for a heteroclinic connection to flip flops.

- $\alpha_{+}<\alpha$ : We lose only our $0 \rightarrow 1$ stationary profiles, as both the local maximum and minimum of $g$ now lie in the interval $(-1,1)$ and so our previously discussed heteroclinic connections corresponding to these two states disappear. 


\subsubsection{Linear Wave Speeds}

Applying precisely the same techniques used in Section 3.1.3, we find an expression for the linear wave speeds associated with our 0 equilibrium,

$$
c=\frac{1+\alpha}{W\left(\frac{-1-\alpha}{e \alpha}\right)} .
$$

The resulting phenomena completely parallel the case of a fold. For $\alpha<-1$, equation (3.2) has two solutions, $c_{-1}$ and $c_{0}$; when $\alpha=-1$, i.e. at the moment at which we gain the existence of a $0 \rightarrow 1$ stationary profile, $c_{-1}$ becomes 0 , and our instability becomes absolute as opposed to convective. Finally, as $\alpha \rightarrow 0, c_{0} \rightarrow 0$, at which point a frustrated front emerges, and instabilities about 0 no longer propagate through our network. Again, the asymptotics near $c=0$ imply a steepening of the invasion fronts near the frustrated regime.

\section{Cusp}

Keeping the methods and results of the previous sections in mind, we now move to study our final nonlinear dynamics, which will now mimic a rescaled cusp bifurcation with codimension 2. Throughout this section, we will work with the following cubic nonlinearity $f(u)=u(1-u)(u-a)$, where we will restrict to $0<a<\frac{1}{2}$.

The remainder of this section is organized as follows. We begin with a discussion of the various period-two equilibria of our system, which we will take throughout this section to be of semi-infinite length. We will then describe various stationary profiles which exist in our network. During this discussion, we will see that for our semi-infinite network the unique dynamics of our $j=0$ cell given from (1.1) will play an important role in the characterization of certain stationary profiles.

We will, throughout this section, drop the Lemma-Proof nature of the previous sections, and instead give a more general overview of the methods used to construct the previously mentioned figures. The bifurcation diagrams in Figure 6 can be constructed using computer algebra, the existence conditions for heteroclinics are the necessary conditions from Lemma 2.6. We did not attempt to systematically verify sufficient conditions as given in Remark 2.11, but found those to hold wherever we verified them by inspection of the graph of the map.

\subsection{Existence and Stability of Equilibria}

Here, we discuss all period-two equilibria of (1.1) and their stability, the results of which are summarized in Figures 6 and 7. Since finding the homogeneous equilibria of our system remains a trivial task, we immediately find the existence of three homogeneous equilibria, $0, a$, and 1 . We find explicit relations between $a$ and $\alpha$ which impart temporal stability to our equilibria, which are summarized below.

- 0 is temporally stable provided $\alpha<\frac{a}{2}$;

- $a$ is always temporally unstable, though as $\alpha$ increases past $\alpha=\frac{a^{2}-a}{2}$, the period-two Morse index of this equilibrium changes from 1 to 2 ;

- 1 is temporally stable provided $\alpha<\frac{1-a}{2}$; 
Of particular importance are the points in $(a, \alpha)$ space at which our various homogeneous equilibria lose their temporal stability (or when the Morse index of our equilibria changes). These are precisely the points associated with the locations of the pitchfork bifurcations shown in Figure 6, and the lines in Figure 7. These bifurcation points will further correspond precisely to the bounds of existence for the flip flop equilibria.

We find in addition to our homogeneous equilibria up to three flip flops. Lacking an explicit expression for the values of these equilibria, we will simply describe them in terms of the curves in Figure 6 as follows:

- Our first flip flop equilibrium, $u_{\mathrm{flip}_{1}}, u_{\mathrm{flop}_{1}}$, corresponds to the two outermost curves (the lower of which will correspond to $u_{\mathrm{flop}_{1}}$, the higher to $\left.u_{\mathrm{flip}_{1}}\right)$. This flip flop is temporally stable over its entire interval of existence, $\alpha_{\text {saddle }}(a)<\alpha$, where $\alpha_{\text {saddle }}(a)$ denotes the location of the saddle-node bifurcation in our system. In future sections, any stationary interface using the symbol $F F$ will refer to this equilibrium.

- We define $u_{\mathrm{flip}_{2}}, u_{\mathrm{flop}_{2}}$ to be the equilibrium given by the two lower curves, which are always temporally unstable, and exist for $\frac{a^{2}-a}{2}<\alpha<\frac{a}{2}$.

- Our final flip flop equilibrium $u_{\mathrm{flip}_{3}}, u_{\mathrm{flop}_{3}}$ corresponds to the two remaining curves, and is always unstable. It exists for $\alpha_{\text {saddle }}(a)<\alpha<\frac{1-a}{2}$.

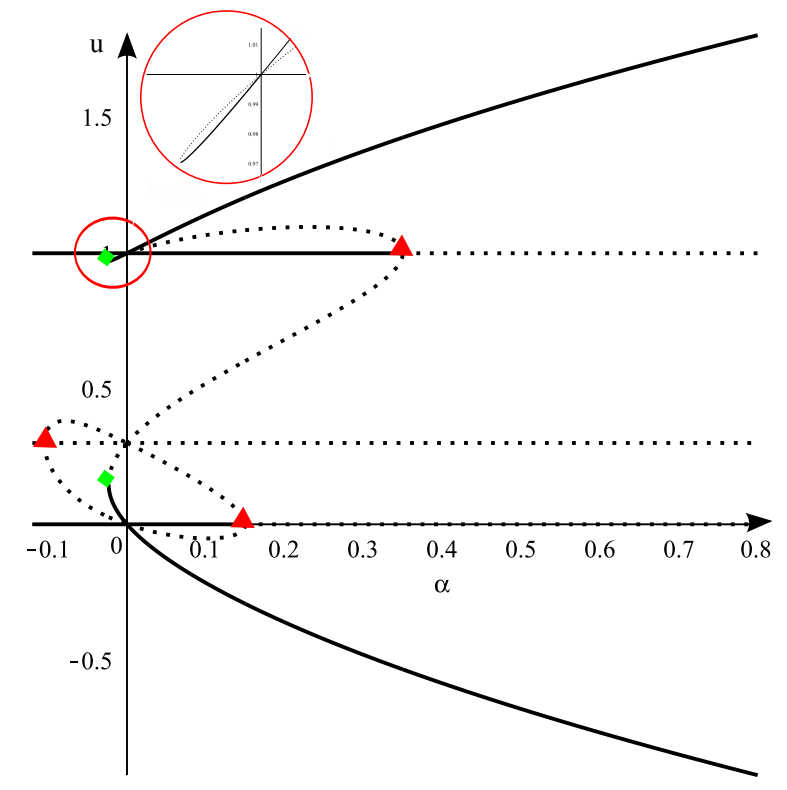

(a) $a=.3$

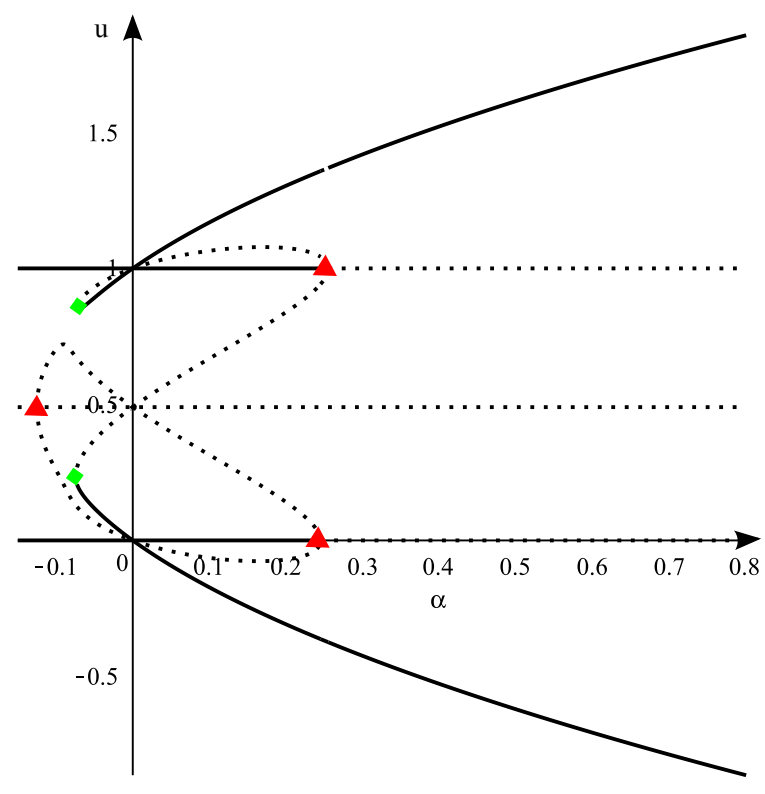

(b) $a=.49$

Figure 6: Bifurcation diagram in the $(a, \alpha)$ plane of the period-two equilibria of our system. Similarly to Figure 3, dotted lines represent temporally unstable equilibria, while solid represent stable equilibria. Triangles correspond to the pitchfork bifurcations associated with our homogeneous equilibria, and diamonds correspond to the saddle node bifurcation. Observe that, at $a=\frac{1}{2}$ we recover Figure 3.

To complete the picture, it now remains to describe how we computed the previously mentioned $\alpha_{\text {saddle }}(a)$, the location of the saddle node bifurcation from which our first flip flop equilibrium emerges. To this end we note that upon adding, and respectively subtracting, the nullclines corre- 


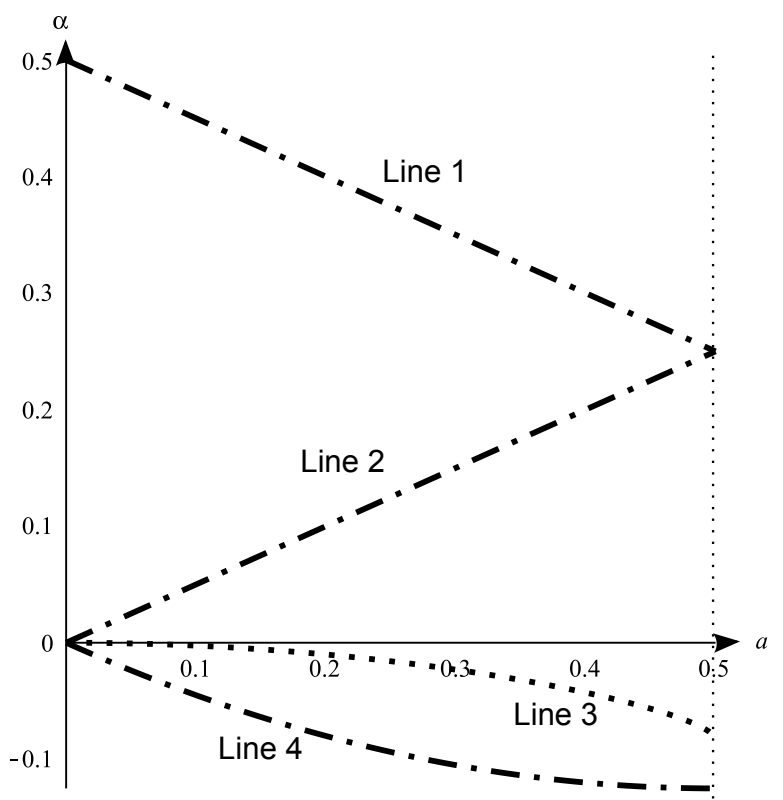

Figure 7: Locations of the various bifurcations in the $a-\alpha$ plane. Here, Line $1=\frac{1}{2}(1-a)$ corresponds to the location of the pitchfork bifurcation associated with the homogeneous equilibrium 1 . Line $2=\frac{a}{2}$ corresponds to the pitchfork associated with 0 . Line 3 shows $\alpha_{\text {saddle }}(a)$. Line $4=\frac{a^{2}-a}{2}$ gives the location of our final pitchfork bifurcation, associated with the equilibrium $a$.

sponding to equations (2.5), we find

$$
\begin{aligned}
& f\left(u_{0}\right)+f\left(u_{1}\right)=0, \\
& \frac{f\left(u_{0}\right)-f\left(u_{1}\right)}{u_{0}-u_{1}}=-2 \alpha .
\end{aligned}
$$

With these equations in mind, we set $u_{0}=u_{\mathrm{flip}_{1}}, u_{1}=u_{\mathrm{flop}_{1}}$, and will now make use of a geometric intuition to find the location of our saddle node. From Figure 6, we see that for $\alpha$ large, $u_{\mathrm{fli}_{1}} \gg 0$ while $u_{\mathrm{flop}_{1}} \ll 0$. As $\alpha$ decreases, we see that $u_{\mathrm{flop}_{1}}$ briefly becomes 0 precisely as $u_{\mathrm{fli}_{1}}$ becomes $_{\mathrm{f}}$ 1. Finally, as $\alpha$ continues to decrease, $u_{\mathrm{fli}_{1}}$ attains its minimum value just as $u_{\mathrm{flop}_{1}}$ attains its maximum value.

Keeping this information in mind we then turn our attention to Figure 8. From (4.1), we see that the images under $f$ of $u_{\mathrm{flip}_{1}}$ and $u_{\mathrm{flop}_{1}}$ must be equal but opposite in sign. Starting at $\alpha=0$, i.e. when $u_{\text {flop }_{1}}=0$ and $u_{\mathrm{fli}_{1}}=1$ and slowing decreasing $\alpha$, we see that the slope of the secant line between our two points must become slowly more positive, attaining its maximum precisely at the location of our saddle node. By visual inspection, we see that this occurs precisely when

$$
u_{\mathrm{flop}_{1}}=\frac{1+a-\sqrt{a^{2}-a+1}}{3}=u_{m i n},
$$

where $u_{\min }$ denotes the minimum of $f$ on the interval $(0,1)$. This constraint may then be used in conjunction with (4.1) to produce a relation $\alpha_{\text {saddle }}(a)$, giving the location of our saddle node bifurcation for all $a$.

Remark 4.1. Directly related to our analysis of period-two equilibria in the cusp setting is the interesting study of Brucal-Hallare and Van Vleck [2] on lattice Nagumo equations with a cusp 


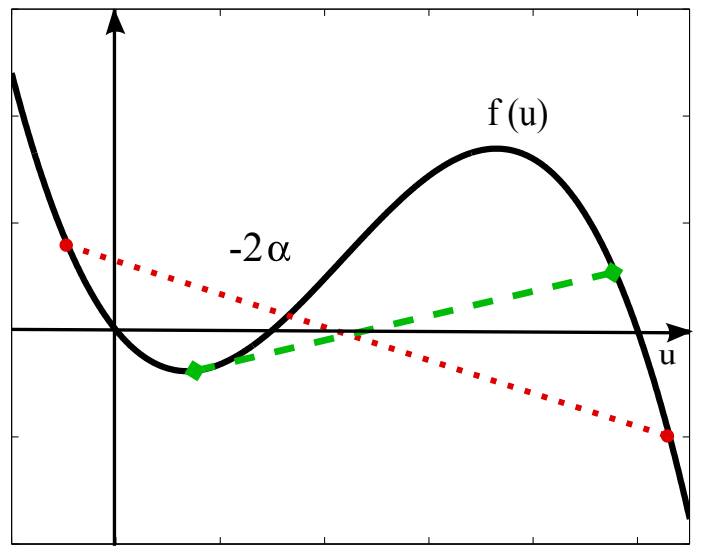

Figure 8: Illustrative example using the geometric argument for computing $\alpha_{\text {saddle }}(a)$. Each secant line joints the image of $u_{\mathrm{flip}_{1}}$ with that of $u_{\mathrm{flop}_{1}}$ under $f$. We see that the red dotted line, which has negative slope, corresponds to positive $\alpha$, while the green dash-dot line with positive slope corresponds to $\alpha$ negative.

nonlinearity. The coupling in their case is bi-directional, symmetric, nearest-neighbor coupling. In particular, they consider negative linear diffusion, which corresponds to our case $\alpha>0$, and classify all possible period-two equilibria for (4.1) with precise numbers of equilibria for a wide range of parameter values. Those results agree with our Figure 6. Much of the information in Figure 7 is in fact contained in [2, Figure 1] but reproduced here for convenience. Of course, the analysis of equilibrium profiles in the following section is entirely different from [2] because of the nature of the coupling.

\subsection{Stationary Profiles}

Having looked at the stability of our equilibria, we now analyze existence conditions of stationary interfaces of forms $0 \rightarrow F F, 1 \rightarrow F F, 0 \rightarrow 1,1 \rightarrow 0, F F \rightarrow 1, F F \rightarrow 0$, and $F F \rightarrow F F^{\prime}$. Here $F F \rightarrow F F^{\prime}$ designates a cell system of flip flops with a defect, e.g. a system that goes flip flop flip flip flop or flop flip flop flop flip. Similarly to previous sections, we make use of Remark 2.9 to establish boundaries in the $a, \alpha$ in which heteroclinics, and thus stationary profiles, exist between our two states in question. While the tedious details of this analysis will not be given here, upon inspecting the map in detail, one can in fact verify that our boundaries are sharp. Our results are given in Figure 9 and Table 1. In the following section, we compare these predictions to numerical results.

To illustrate those results, consider Regions 1 and 2. In Region 1, Table 1 shows that there exist only two stationary interfaces, $0 \rightarrow F F$ and $1 \rightarrow F F$. We now examine changes as we cross the boundary from Region 1 into Region 2. Referencing Table 1 and looking at both the rows for Region 1 and Region 2, we see that the only change is for the interface $0 \rightarrow 1$, which exists in Region 2 but not in Region 1. One can examine all region boundaries in this fashion, obtaining a complete bifurcation diagram for stationary interfaces.

Remark 4.2. We find that only the boundary between Region 5 and Region 8 and the boundary between Regions 6 and 7 can be expressed explicitly as $-\left(\frac{a}{2}\right)^{2}$ and $-\left(\frac{1-a}{2}\right)^{2}$, respectively. In fact, Regions 8 and 9 are subregions of Regions 6 and 7 , respectively. In these subregions, the same 


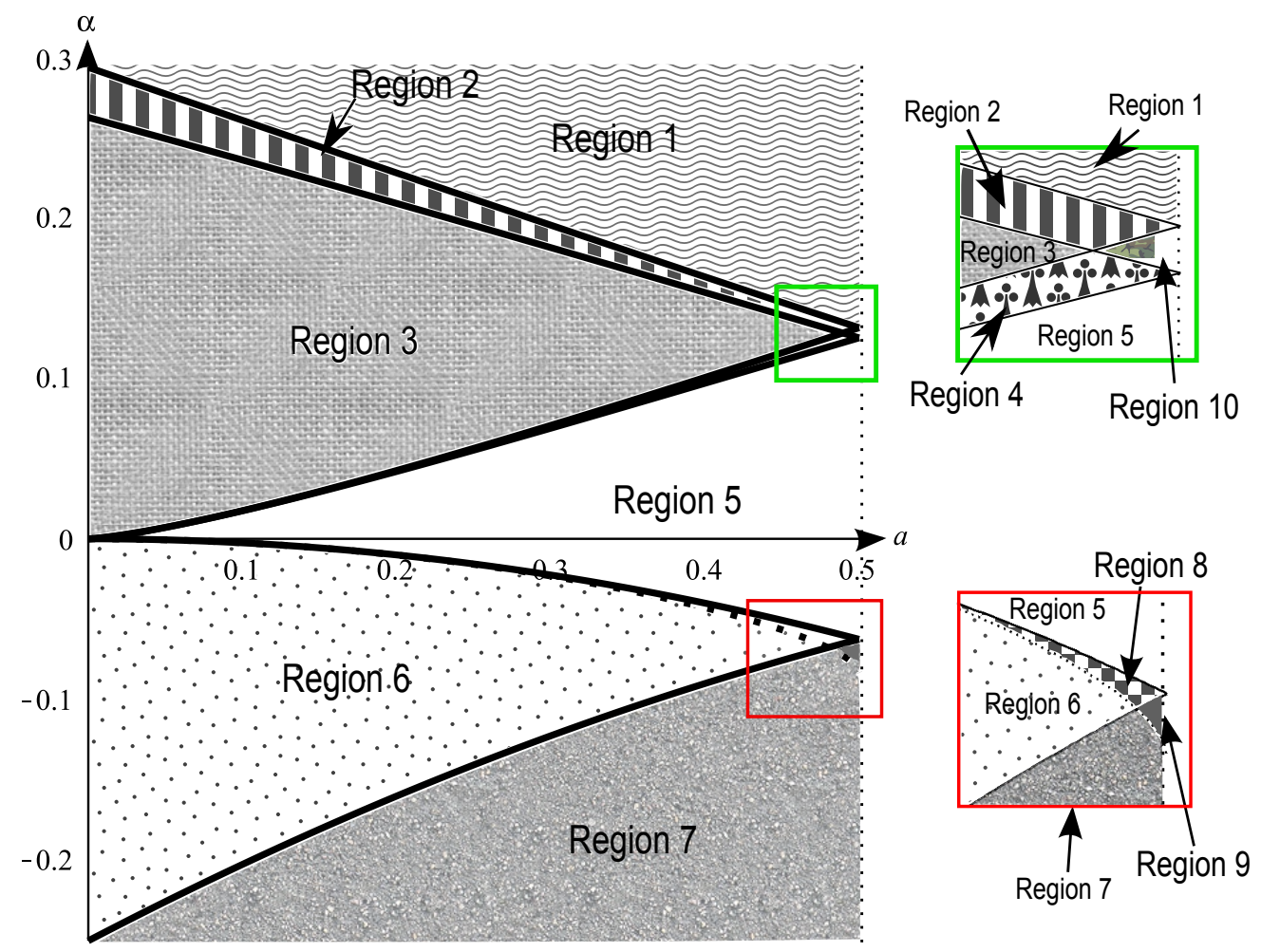

Figure 9: Each solid line expresses $\alpha$ as a function of $a$ and each one represents the upper bounds for the existence of a stationary interface. The dotted line expresses the value of $\alpha$ for which a saddle node bifurcation occurs. For each region the existence of a given interface can be found in Table 1.

\begin{tabular}{|c|c|c|c|c|c|c|c|}
\hline Region & $0 \rightarrow F F$ & $1 \rightarrow F F$ & $0 \rightarrow 1$ & $1 \rightarrow 0$ & $F F \rightarrow 1$ & $F F \rightarrow 0$ & $F F \rightarrow F F^{\prime}$ \\
\hline 1 & $\mathrm{X}$ & $\mathrm{X}$ & $\mathrm{N}$ & $\mathrm{N}$ & $\mathrm{N}$ & $\mathrm{N}$ & $\mathrm{N}$ \\
\hline 2 & $\mathrm{X}$ & $\mathrm{X}$ & $\mathrm{X}$ & $\mathrm{N}$ & $\mathrm{N}$ & $\mathrm{N}$ & $\mathrm{N}$ \\
\hline 3 & $\mathrm{X}$ & $\mathrm{X}$ & $\mathrm{X}$ & $\mathrm{N}$ & $\mathrm{X}$ & $\mathrm{N}$ & $\mathrm{X}$ \\
\hline 4 & $\mathrm{X}$ & $\mathrm{X}$ & $\mathrm{X}$ & $\mathrm{X}$ & $\mathrm{X}$ & $\mathrm{N}$ & $\mathrm{X}$ \\
\hline 5 & $\mathrm{X}$ & $\mathrm{X}$ & $\mathrm{X}$ & $\mathrm{X}$ & $\mathrm{X}$ & $\mathrm{X}$ & $\mathrm{X}$ \\
\hline 6 & $\mathrm{NA}$ & $\mathrm{NA}$ & $\mathrm{X}$ & $\mathrm{N}$ & $\mathrm{NA}$ & $\mathrm{NA}$ & $\mathrm{NA}$ \\
\hline 7 & $\mathrm{NA}$ & $\mathrm{NA}$ & $\mathrm{N}$ & $\mathrm{N}$ & $\mathrm{NA}$ & $\mathrm{NA}$ & $\mathrm{NA}$ \\
\hline 8 & $\mathrm{X}$ & $\mathrm{N}$ & $\mathrm{X}$ & $\mathrm{N}$ & $\mathrm{T}$ & $\mathrm{T}$ & $\mathrm{T}$ \\
\hline 9 & $\mathrm{~N}$ & $\mathrm{~N}$ & $\mathrm{~N}$ & $\mathrm{~N}$ & $\mathrm{~T}$ & $\mathrm{~T}$ & $\mathrm{~N}$ \\
\hline 10 & $\mathrm{X}$ & $\mathrm{X}$ & $\mathrm{X}$ & $\mathrm{X}$ & $\mathrm{N}$ & $\mathrm{N}$ & $\mathrm{N}$ \\
\hline
\end{tabular}

Table 1: Existence of stationary interfaces: $\mathrm{X}$ indicates that the stationary interface exists, $\mathrm{N}$ indicates that the stationary interface does not exist, NA indicates that we cannot examine the interface because stable flip flops no longer exist in the region, and $\mathrm{T}$ indicates that the stationary interface exists in the infinite but possibly not in the semi-infinite lattice; see Section 4.4 for more details on this case.

behavior is observed for the interfaces $0 \rightarrow 1,1 \rightarrow 0$; however, since stable flip flops still exist in these subregions we distinguish them as separate regions in order to examine the interfaces $0 \rightarrow F F$, $1 \rightarrow F F, F F \rightarrow 1, F F \rightarrow 0$, and $F F \rightarrow F F^{\prime}$. The line specifying the boundary between each of these regions and their subregions is given by the flip flop saddle node. 


\subsection{Numerical Simulations}

In this section, we display numerical simulations exhibiting interface dynamics for various regions of Figure 9. These simulations confirm that the necessary information gathered in Figure 9 and Table 1 give sharp criteria. In all the simulation figures of this section the notation $R_{A} / R_{B}$ specifies that we are looking at region $A$ near region $B$. Time is increasing vertically, and the horizontal axis of each picture is the cell index $j$ where $1 \leq j \leq 40$. In addition, for all simulation figures lighter colors correspond to values near 1, while darker colors correspond to values near 0 . Note however that the color scaling is not uniform across figures.

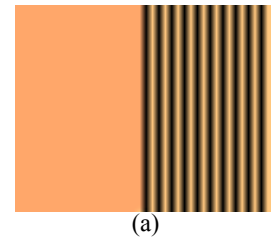

(a)

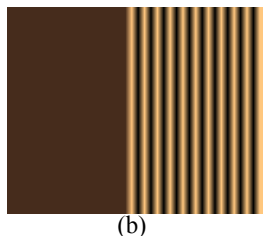

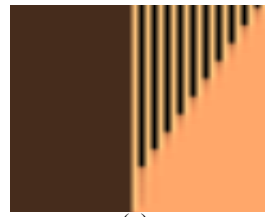

(c)

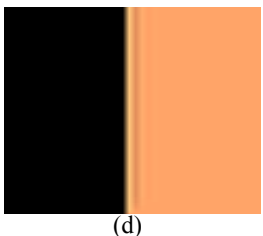

(d)

Figure 10: (a): $R_{1}, 1 \rightarrow F F, a=0.3, \alpha=0.197$, and $t=300$ units. (b): $R_{1}, 0 \rightarrow F F, a=0.3, \alpha=0.197$, and $t=300$ units. (c): $R_{1} / R_{2}, 0 \rightarrow 1, a=0.3, \alpha=0.197$, and $t=300$ units. (d): $R_{2} / R_{1}, 0 \rightarrow 1, a=0.3$, $\alpha=0.192$, and $t=300$ units.
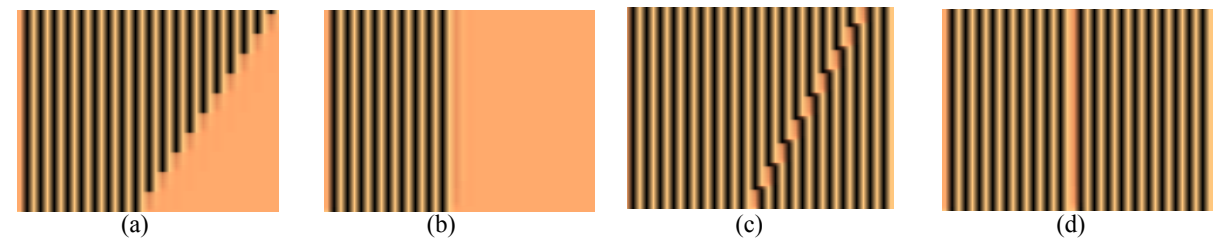

Figure 11: (a): $R_{2} / R_{3}, F F \rightarrow 1, a=0.3, \alpha=0.183$, and $t=800$ units. (b): $R_{3} / R_{2}, F F \rightarrow 1, a=0.3$, $\alpha=0.18$, and $t=300$ units. (c): $R_{2} / R_{3}, F F \rightarrow F F^{\prime}, a=0.3, \alpha=0.183$, and $t=800$ units. (d): $R_{3} / R_{2}$, $F F \rightarrow F F^{\prime}, a=0.3, \alpha=0.18$, and $t=300$ units.
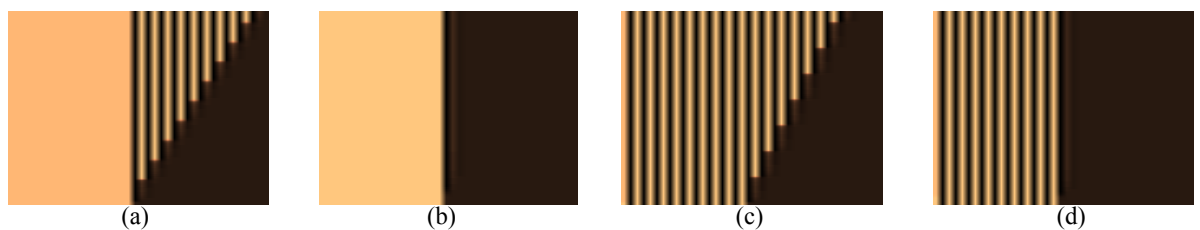

Figure 12: (a): $R_{3} / R_{4}, 1 \rightarrow 0, a=0.3, \alpha=0.072$, and $t=800$ units. (b): $R_{4} / R_{3}, 1 \rightarrow 0, a=0.3$, $\alpha=0.07$, and $t=300$ units. (c): $R_{4} / R_{5}, F F \rightarrow 0, a=0.3, \alpha=0.07$, and $t=1000$ units. (d): $R_{5} / R_{4}$, $F F \rightarrow 0, a=0.3, \alpha=0.068$, and $t=300$ units.

\subsection{Effects of Truncation}

In this section we will briefly discuss the effects of the $0^{\text {th }}$ cell in a semi-infinite system and discuss Figure 16. More precisely, upon examining (1.1) one can see that if the $0^{\text {th }}$ cell is initialized to $u_{*}$ such that $f\left(u_{*}\right) \neq 0$, the cell will change in value until equal to the nearest homogeneous equilibrium $\bar{u}$, e.g. 1 or 0 .

In numerical simulations, we expect a stationary $F F \rightarrow A$ interface in certain regions, though these interfaces may in fact appear to be non-stationary when studied in a semi-infinite lattice (see the 

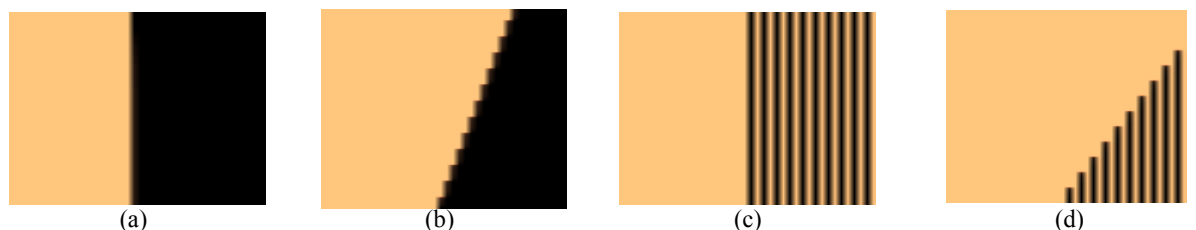

(d)

Figure 13: (a): $R_{5} / R_{8}, 1 \rightarrow 0, a=0.3, \alpha=-0.022$, and $t=300$ units. (b): $R_{8} / R_{5}, 1 \rightarrow 0, a=0.3$, $\alpha=-0.023$, and $t=2000$ units. (c): $R_{5} / R_{8}, 1 \rightarrow F F, a=0.3, \alpha=-0.022$, and $t=300$ units. (d): $R_{8} / R_{5}, 1 \rightarrow F F, a=0.3, \alpha=-0.023$, and $t=1500$ units.
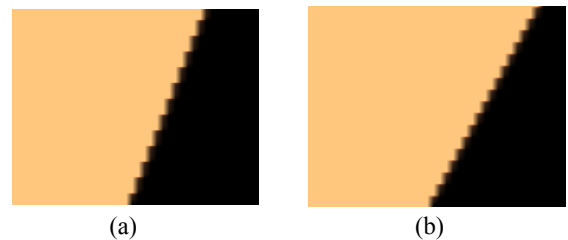

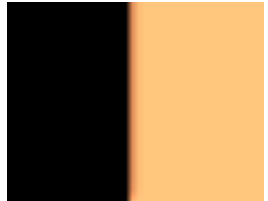

(c)

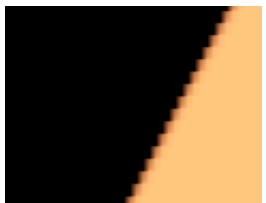

(d)

Figure 14: (a): $R_{8} / R_{6}, 1 \rightarrow 0, a=0.3, \alpha=-0.022$, and $t=2000$ units. (b): $R_{6} / R_{8}, 1 \rightarrow 0, a=0.3$, $\alpha=-0.024$, and $t=1500$ units. (c): $R_{6} / R_{7}, 0 \rightarrow 1, a=0.3, \alpha=-0.121$, and $t=300$ units. (d): $R_{7} / R_{6}$, $0 \rightarrow 1, a=0.3, \alpha=-0.124$, and $t=2000$ units.

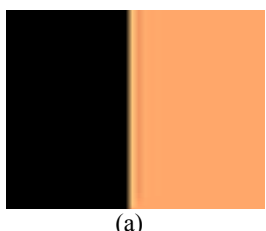

(a)

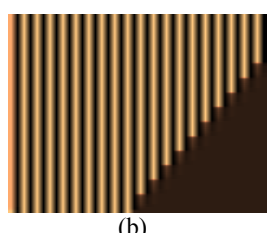

(b)

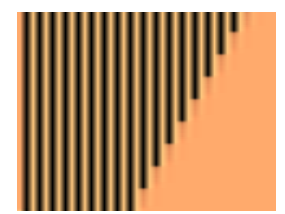

(c)

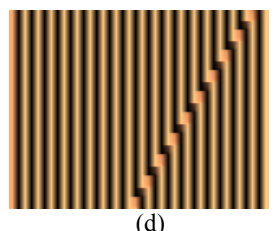

(d)

Figure 15: (a): $R_{10}, 0 \rightarrow 1, a=0.496, \alpha=0.128$, and $t=300$ units. (b): $R_{10}, F F \rightarrow 0, a=0.496$, $\alpha=0.128$, and $t=800$ units. (c): $R_{10}, F F \rightarrow 1, a=0.496, \alpha=0.128$, and $t=800$ units. (d): $R_{10}$, $F F \rightarrow F F^{\prime}, a=0.496, \alpha=0.128$, and $t=1500$ units.

entries ' $\mathrm{T}$ ' of Table 1). This can be explained by the behavior of the $0^{\text {th }}$ cell creating a new interface which may be non-stationary. For example, in Region 8 of Figure 9 , one would expect the $F F \rightarrow 1$ interface to be stationary; however, if the $0^{\text {th }}$ cell of the $F F$ cluster is initialized to the flip, the interface will appear non-stationary because the $0^{\text {th }}$ cell goes from a value of flip to a value of 1 creating a $1 \rightarrow F F$ interface which is non-stationary as seen in Table 1 . This causes an invasion front to propagate through the system during the simulation making the original interface appear non-stationary.

In Figure 16, (a) and (e) illustrate the effect of the $0^{\text {th }}$ node on a $F F \rightarrow F F^{\prime}$ interface. They also show how the two different $F F \rightarrow F F^{\prime}$ interfaces possess different properties. Figures (b), (c), and (d) all demonstrate boundary effects on the $F F \rightarrow 0$ interface. In Region 8, an effect similar to (c) occurs, but is omitted here. Similarly, (f), (g), and (h) demonstrate the boundary effects on the $F F \rightarrow 1$ interface. Again in Region 8 an effect similar to (g) occurs, but is omitted here. On the other hand, figures (d) and (h) showcase effects unique to Region 9, whose their corresponding counterparts in Region 8 are shown in (b) and (f), respectively. 


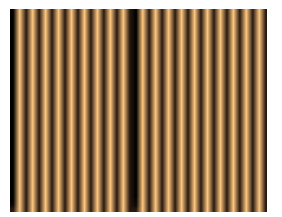

(a)

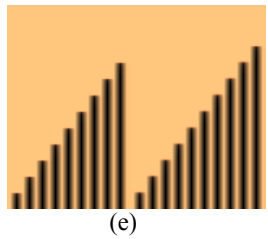

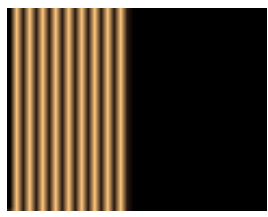

(b)

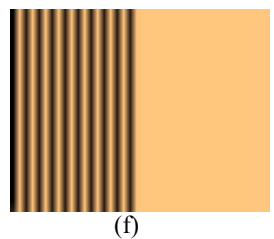

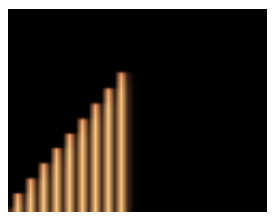

(c)

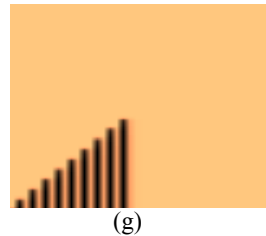

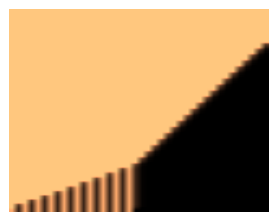

(d)

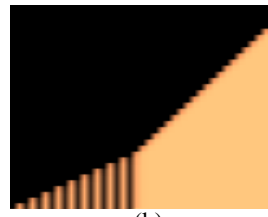

(h)

Figure 16: (a): $R_{8}, F F \rightarrow F F^{\prime}, a=0.3, \alpha=-0.023$, and $t=300$ units. (b): $R_{8}, F F \rightarrow 0, a=0.3$, $\alpha=-0.023$, and $t=300$ units. (c): $R_{9}, F F \rightarrow 0, a=0.493, \alpha=-0.069$, and $t=800$ units. (d): $R_{9}$, $F F \rightarrow 0, a=0.493, \alpha=-0.069$, and $t=1500$ units. (e): $R_{8}, F F \rightarrow F F^{\prime}, a=0.3, \alpha=-0.023$, and $t=1500$ units. (f): $R_{8}, F F \rightarrow 1, a=0.3, \alpha=-0.023$, and $t=1500$ units. (g): $R_{9}, F F \rightarrow 1, a=0.493$, $\alpha=-0.069$, and $t=800$ units. (h): $R_{8}, F F \rightarrow 1, a=0.493, \alpha=-0.069$, and $t=2000$ units.

\section{Discussion}

Here we have studied coherent structures in feed-forward chains quite systematically. We expect that our results can be easily adapted to other nonlinearities $f$. While our previously discussed "toy nonlinearities" are motivated by simple normal forms at individual lattice sites, it would be interesting to obtain these normal forms in a more systematic way using ideas from [10].

On the other hand, it would be interesting to explore more complicated cell dynamics. We expect some of our results to survive quite literally. For instance, we expect that bifurcations in finite lattices can still be viewed as transitions from convective to absolute instabilities, corresponding to the formation of infinitely steep, frustrated invasion fronts. Further, we expect that in certain cases these complex systems may still be understood through their spatial dynamics in $j$, i.e. by reduction to interval maps. As an example, we mention excitable dynamics,

$$
\begin{aligned}
& \dot{u}_{j}=f\left(u_{j}\right)-v_{j}+\alpha\left(u_{j}-u_{j-1}\right), \\
& \dot{v}_{j}=\varepsilon\left(u_{j}-\gamma v_{j}\right),
\end{aligned}
$$

where stationary interfaces solve a simple scalar recursion after substituting $v_{j}:=u_{j} / \gamma$ into $f\left(u_{j}\right)-$ $v_{j}+\alpha\left(u_{j}-u_{j-1}\right)=0$. One can then study the effects of front pinning on excitation pulses in a systematic fashion.

Similarly, Hopf normal form dynamics

$$
\dot{u}_{j}=u_{j} f\left(\left|u_{j}\right|^{2}\right)+\alpha\left(u_{j}-u_{j-1}\right), \quad u_{j} \in \mathbb{C},
$$

can be reduced to iterations for $\left|u_{j}\right| \in \mathbb{R}$, choosing relative phase $\operatorname{shifts} \arg \left(u_{j}-u_{j-1}\right)$ appropriately.

\section{Acknowledgments}

This research was conducted during Summer 2014 in the REU: Complex Systems at the University of Minnesota Department of Mathematics, funded by the National Science Foundation (DMS1311414) and (DMS-1311740); see (http://math.umn.edu/ gfaye/reu.html). 


\section{References}

[1] A.N. Bers. Space-time evolution of plasma instabilities - absolute and convective. In: Rosenbluth, M.N., Sagdeev, R.Z. (eds.), Handbook of Plasma Physics, 1983.

[2] M. Brucal-Hallare and E. Van Vleck. Traveling Wavefronts in an Antidiffusion Lattice Nagumo Model. SIAM J. Appl. Dyn. Syst., vol. 10, no.3, pp. 921-959, 2011.

[3] A. Couairon and J-M. Chomaz. Absolute and convective instabilities, front velocities and global modes in nonlinear systems. Physica D, 108, pp. 236-276, 1997.

[4] R.M. Corless, G.H. Gonnet, D.E.G. Hare, D.J. Jeffrey and D.E. Knut. On the Lambert $W$ function. Advances in Computational Mathematics, 5, pp 329-359, 1996.

[5] R. Goh and A. Scheel. Triggered fronts in the complex Ginzburg-Landau equation. J. Nonlinear Sc., 24, issue 1, pp. 117-144, 2014.

[6] M. Golubitsky and I. Stweart. Nonlinear dynamics of networks: the groupoid formalism. Bull. Amer. Math. Soc., 43, pp. 305-364, 2006.

[7] A. Hoffman and B. Kennedy. Existence and uniqueness of traveling waves in a class of unidirectional lattice differential equations. $D C D S-A, 30$, pp. 137-167, 2011.

[8] M. Holzer and A. Scheel. Criteria for Pointwise Growth and Their Role in Invasion Processes. J. Nonlinear Sci., 24, issue 4, pp. 661-709, 2014.

[9] B. Rink and J. Sanders. Amplified Hopf bifurcations in feed-forward networks. SIAM J. Appl. Dyn. Syst., 12, pp. 1135-1157, 2013.

[10] B. Rink and J. Sanders. Coupled cell networks: semigroups, Lie algebras and normal forms. Trans. Amer. Math. Soc., in press, 2014.

[11] W. van Saarloos. Front propagation into unstable states. Physics Reports, 386, pp 29-222, 2003.

[12] B. Sandstede and A. Scheel. Absolute and convective instabilities of waves on unbounded and large bounded domains. Physica D, 145, pp. 233-277, 2000.

[13] O.M. Sharkov'ski. Co-Existence of Cycles of a Continuous Mapping of a Line onto Itself. Ukranian Math. Z., 16, pp. 61-71, 1964. 